\title{
Vostra eccellenza
}

Giovanni Lodi

Dipartimento di Scienze Biomediche, Chirurgiche e Odontoiatriche

Università degli Studi di Milano

via Beldiletto 1/3 Milano 20142

Italia

giovanni.lodi@unimi.it

tel. + 390250319021

fax. +390250319041

Per i pochi che non l'avessero notato, faccio presente che oggi essere un buon odontoiatra, anzi un ottimo odontoiatra, proprio non è sufficiente.

Ci vuole l'eccellenza.

Eccellenza è una tra le parole più usate quando si parla di odontoiatria e medicina: trattamenti di eccellenza, centri di eccellenza, eccellenza clinica, ma anche eccellenza nella didattica e nella ricerca.

Provate a farvi un giro su Google e scoprirete che l'eccellenza nel nostro campo è dappertutto, dal grande centro universitario, allo studio mono professionale, fino alla catena in franchising.

Ma cosa sarà mai questa eccellenza?

È un manufatto che proiettato su uno schermo grande quanto un campo da tennis non mostra difetti? È il sorriso smagliante di una persona bellissima? È un centro immacolato che ti accoglie con i sorrisi di cui sopra? Non è facile, almeno per me, immaginare cosa ci sia o cosa ci dovrebbe essere dietro questa parola.

Ma visto che di parola si tratta, ricorro al dizionario. Non ho sotto mano il mio vecchio Devoto Oli e quindi ne consulto uno online, dove leggo che con eccellenza si deve intendere: "qualità di persona o cosa che eccelle; superiorità assoluta, altissimo grado di perfezione".

Però, mica male, vi eravate accorti di tutta questa perfezione? E dire che qualcuno affermava che la perfezione non è di questo mondo.

In realtà, però, non abbiamo fatto molta strada nella nostra ricerca del senso dell'eccellenza.

Perché la perfezione in medicina è davvero possibile? E chi la stabilisce? E poi, se spostiamo il punto di vista, una cosa perfetta non è migliorabile. Lo direste per l'odontoiatria e più in generale per la medicina? lo proprio no.

O forse è solo una parola, come per i campionati di calcio, dove l'eccellenzaviene dopo la serie D.

Buona lettura 